we have hitherto gone the death-rate will, then, appear to be upwards of ten per cent. of those in whom syphilization has been attempted or practised. We have a right to conclude that these patients were syphilitic, and that they had taken no mercury -an indication at once that some of the worst forms of syphilis occur in those who have not been subjected to mercurial treatment, and that some forms of syphilis at least are not benefited by syphilization. I would not, however, for a moment imply that the treatment pursued had any connexion with the death of these patients.

6. Mr. Lane thinks that it would have been better if I had obtained the "authentic" particulars of the facts which I have stated through him. I think otherwise. When evidence derived from different sources points to the same conclusion, it is much stronger than when it comes through one channel only; and when the evidence thus obtained in various ways would appear to point to different results, the points of difference stand out in much bolder relief than if they have been given by the same person. For instance, had I received my information only from Mr. Lane, I should have believed that Dr. Boeck had "undoubtedly" succeeded in producing a lineal series of inoculations from an uncomplicated indurated sore; as it is, I very much doubt the fact. The secretion from the sore in this case was inoculated by Dr. Boeck, Mr. Coulson, and myself without result. The patient was then inoculated with some matter from another patient; and it was not till after he had been so inoculated, as he distinctly informed me, that the matter from his own sore became inoculable on himself. As explained in my lecture, the same lancet was sometimes used (not by Mr. Coulson) in the attempts to inoculate this patient with the secretion of his own sore and the matter from another patient.

As the fact is now brought very prominently forward by Mr. Lane's letter that this is the only case in which it was supposed that Dr. Boeck had inoculated an indurated chancre, the profession may like to know a little more about it; and I therefore beg to forward you the following note from Mr. Coulson. I am, Sir, your obedient servant,

Savile-row, May 1st, 1866.

$$
\text { [C O P Y.] }
$$

Henry Lee.

DEAR Mr. LEE, - In answer to the question contained in your note of the 28th, I beg to inform you that Dr. Boeck did not succeed in inoculating $\mathrm{D}$ - from his own indurated chancre. The first two inoculations on this patient were made by Dr. Boeck on Nov. 27th. These both failed, and were the only inoculations he made. I continued the inoculations, and obtained a positive result at the end of three weeks, after making two inoculations daily during the whole time of the period, apparently without effect.

Believe me, yours very truly,

St. James's-place, April 30th, 1866 . WALTER J. COULSON.

\section{SUBCUTANEOUS INJECTIOON OF MERCURY IN CONSTITUTIONAL SYPHILIS.}

\section{To the Editor of The Laxcet.}

Sir,-The paragraph in your journal of April 7th on Dr. Scarenzio's experiments in injecting mercury subcutaneously for treatment of constitutional syphilis induces me to narrate the results of some experiments made by myself, in 1864 and 1865 , on the same method of employing mercury in syphilis. My experiments were extended to eleven individuals, some afflioted with the earlier, some with the later forms of the constitutional affection. I selected the bichloride dissolved in water, thinking that I could more accurately measure its absorption than that of any other form of mercury; and $I$ found its effect to be quite as active as that of any of the rest when given by the mouth. For my injections I used Coxeter's hypodermic syringe, introducing ordinarily six minims of the solution.

My first case was a girl in Mr. Erichsen's ward in University College Hospital, that gentleman having kindly permitted me to employ this method of mercurializing on such of his hospital patients for whom mercury was prescribed. I injected in this case, night and morning, one-tenth of a grain of the bichloride, inserting the canula through the skin of the arm or shoulder. By the time eight-tenths of a grain were introduced the gums were swollen and painful, the patient complained much of soreness in the mouth \&c., and the breath had become distinctly mercurial in odour. This patient, impatient of the pain caused by the prick of the syringe, soon declined to submit herself any more to this mode of treatment, and recourse was then had to pills to prolong the required effect of the mercury.

I continued my experiments on other patients from time to time, with the following results:- In four cases mercurializa. tion was produced when eight-tenths of a grain had been in. jected; in four others when a grain and one-fifth, and in one when a grain and one-third, had been introduced.

In the eleventh case I was unable to produce the ordinary effects of mercury in any way. This case was a gentleman with an indurated chancre and enlarged glands of the groin. Before injection was tried with him, he had taken mercury in frequent doses internally, and had used mercurial vapour-baths and inunctions for three months, without any sion of the in. fluence of the medicine than a slight improvement of his symp. toms, enjoying all the time apparently excellent bodily health. That no doubt should exist whether the mercury was really taken, I injected one-eighth of a grain of bichloride night and morning subcutaneously for thirteen days, and on the sixth day he took in addition half a grain of calomel four times a day at the least, being ordered to take it every two hours. The combined result of which was, at the end of a fortnight, to render the gums slightly swollen and sore. Remaining well during four months of mercurial treatment, the induration meanwhile having greatly diminished, the patient grew tired of further physicking, left town for the sea-side, and has en. joyed good health to the present time, a period of ten months. The case is a remarkable example of the resisting power of certain persons to the effects of mercury.

In employing the subcutaneous method, the quantities in. jected at a time varied from one-tenth to one-fourth of a grain. I found that where one-fourth of a grain was injected, the patients complained of griping, purging, and other symptoms of mercurialization, and the site of injection became painful for some hours afterwards. This result was also caused if more than six or eight drops were introduced. It was, moreover, difficult to get in so much, as part would escape at the wound. I found that a sixth or fifth of a grain dissolved in six minims of water to be the most convenient quantity both in bulk and concentration, enabling me to introduce a grain in two days if $I$ repeated the injections thrice a day; and where the gums were slightly swollen they could be so kept by one injection daily of this quantity, while the patient was able to follow his occupation out of doors as well as if he were taking mercury in any other manner.

The other local effects were-1st, the pain of the puncture, which was trifling, and soon subsided, especially if the outer aspect of the limbs was selected; 2nd, a general soreness and tenderness of the skin if the injections were made too closely to each other; and lastly, in one instance inflammation, with pustules at the punctures, though even in this case this amount of irritation could be avoided if the point of the syringe was carried clearly some distance beyond the skin into the cellular tissue.

Hence in all cases, with one exception, mercurialization was produced by very small doses of the drug, and as rapidly, if not more so, than by any other method. In this exception the method failed when others failed also. In employing subcutaneous injections, the main advantage was the small quantity of mercury necessary to produce the desired effect; the main disadvantage, the pain attending the administration of the medicine. In subcutaneous injection of anodynes, on the other hand, the pain of the prick is so trifling compared with the pain to be allayed that the patient remains indifferent to it. I am, Sir, yours, \&c.,

Weymouth-street, April, 1866.

$$
\text { M. BERKELEY Hill, F.R.C.S. }
$$

\section{NEWCASTLE-ON-TYNE.}

(FROM OUR OWN CORRESPONDENT.)

AT the last meeting of our Medical Society a paper was read by Mr. T. A. H. Dodd on a series of cases which occurred in his practice at a farmhouse near Ponteland, a village a few miles from Newcastle. As these cases have occupied the attention of the profession and the public in the North for some time past, and are likely to do so to a far greater and wider extent before long, and may, probably, give rise to many important medico-legal considerations, I here give an outline of Mr. Dodd's very interesting paper. 